INPLASY

PROTOCOL

To cite: Gao et al. Global and Regional Prevalence and Burden for Premenstrual Syndrome and Premenstrual Dysphoric Disorder: A study protocol for systematic review and meta-analysis. Inplasy protocol 2021120065. doi: 10.37766/inplasy2021.12.0065

Received: 13 December 2021

Published: 13 December 2021

Corresponding author: Mingzhou Gao

gmingzhou@163.com

Author Affiliation:

Shandong University of Traditional Chinese Medicine

Support: Shandong medical and health sc.

Review Stage at time of this submission: The review has not yet started.

Conflicts of interest: None declared.

\section{Global and Regional Prevalence and Burden for Premenstrual Syndrome and Premenstrual Dysphoric Disorder: A study protocol for systematic review and meta-analysis}

Gao, M1; Cheng, X2; Zhang, H³; Gao, Z4; Sun, Y5; Qiao, M6; Gao, D? .

Review question / Objective: Estimation of the epidemiological burden of PMS/PMDD can serve as scientific basis for prevention and management of premenstrual disorders.

Condition being studied: Premenstrual syndrome (PMS) and Premenstrual dysphoric disorder (PMDD) are becoming common mental diseases in women impairing daily functioning.Herein, we firstly provide a protocol to perform estimation on the prevalence and risk factors for PMS/PMDD in the general population globally and regionally.

Information sources: Eight databases comprising PubMed, Chinese National Knowledge Infrastructure (CNKI), EMBASE, Chinese Biomedical Literature Database (CBM), Wanfang Database, the Cochrane Central Register of Controlled Trials (Cochrane Library), Web of Science, as well as Chinese VIP Information will be systematically queried -from database inception until April 15, 2022.

INPLASY registration number: This protocol was registered with the International Platform of Registered Systematic Review and Meta-Analysis Protocols (INPLASY) on 13 December 2021 and was last updated on 13 December 2021 (registration number INPLASY2021120065).

\section{INTRODUCTION}

Review question / Objective: Estimation of the epidemiological burden of PMS/PMDD can serve as scientific basis for prevention and management of premenstrual disorders.
Condition being studied: Premenstrual syndrome (PMS) and Premenstrual dysphoric disorder (PMDD) are becoming common mental diseases in women impairing daily functioning.Herein, we firstly provide a protocol to perform estimation on the prevalence and risk 
factors for PMS/PMDD in the general population globally and regionally.

\section{METHODS}

Search strategy: ("Premenstrual Syndrome” [Title/Abstract] OR premenstrual syndrome[MeSH Terms] OR premenstrual syndromes[MeSH Terms] OR premenstrual tension[MeSH Terms] OR syndrome, premenstrual[MeSH Terms] OR Premenstrual dysphoric disorder[Title/ Abstract]) OR Disorder, Premenstrual Dysphoric [MeSH Terms] OR Dysphoric Disorder, Premenstrual [MeSH Terms]ORPremenstrual Dysphoric Syndrome[MeSH Terms] OR Syndrome, Premenstrual Dysphoric[MeSH Terms] AND (prevalence[Title/Abstract] OR prevalence[MeSH Terms] OR analysis, cross sectional[MeSH Terms] OR "Cross sectional"[Title/Abstract] OR Observational[Title/Abstract] OR "Case control"[Title/Abstract] OR Cohort[Title/ Abstract]OR epidemiology [Title/Abstract] OR incidence[Title/Abstract]).

Participant or population: Reproductive age females.

Intervention: None.

\section{Comparator: None.}

Study designs to be included: Populationbased and reported the prevalence of PMS/ PMDD or risk factors for PMS/PMDD.

Eligibility criteria: Included studies had to be population-based and reported the prevalence of PMS/PMDD or risk factors for PMS/PMDD, with any kind of random or nonrandom sampling in reproductive age females, in the common or school-hinged populace, across all ethnicities, educational backgrounds, socioeconomic, as well as living in all countries globally.

Information sources: Eight databases comprising PubMed, Chinese National Knowledge Infrastructure (CNKI), EMBASE, Chinese Biomedical Literature Database (CBM), Wanfang Database, the Cochrane Central Register of Controlled Trials
(Cochrane Library), Web of Science, as well as Chinese VIP Information will be systematically queried - from database inception until April 15, 2022.

Main outcome(s): Prevalence of PMS/ PMDD or risk factors.

Quality assessment / Risk of bias analysis: The STROBE checklist will be utilized for quality evaluation of the enrolled crosssectional researches. Through this evaluation strategy, we will explore six core constituents: study design, outcome data subjects, bias, descriptive, as well as measurement. Study quality of enrolled articles will be independently investigated by two investigators. Disagreements will be reconciled via consents, and third investigator will mediate and reconcile the case, if necessary.

Strategy of data synthesis: A meta-analysis on the prevalence of PMDD will be conducted using STATA V.15.0, and employ the graphical approaches, as well as fixed or random effect models to combine the prevalence estimates. Statistical models will be employed in estimating trends via different research variables including gender, region, and age.

Subgroup analysis: Heterogeneity between the enrolled articles will be explored via the 12 heterogeneity statistic, and subgroup, as well as sensitivity analyses will be applied in assessing the heterogeneity sources.

Sensitivity analysis: Heterogeneity between the enrolled articles will be explored via the 12 heterogeneity statistic, and subgroup, as well as sensitivity analyses will be applied in assessing the heterogeneity sources.

Country(ies) involved: For multi-national authors, inform all the countries involved.

Keywords: systematic review; premenstrual syndrome; premenstrual dysphoric disorder; prevalence.

Contributions of each author:

Author 1 - Mingzhou Gao.

Email: gmingzhou@163.com 
Author 2 - Xunshu Cheng.

Email: c1017532119@qq.com

Author 3 - Hao Zhang.

Email: zhanghaojune@126.com

Author 4 - Zhan Gao.

Email: 1107658885@qq.com

Author 5 - Ya Sun.

Email: xiaojiu9977@qq.com

Author 6 - Mingqi Qiao.

Email: qmingqi@163.com

Author 7 - Dongmei Gao.

Email: gcy_112@163.com 